\title{
Ranking with Uncertain Labels and Its Applications
}

\author{
Shuicheng Yan ${ }^{1}$, Huan Wang ${ }^{2}$, Jianzhuang Liu, Xiaoou Tang ${ }^{2}$, and Thomas S. Huang ${ }^{1}$ \\ ${ }^{1}$ ECE Department, University of Illinois at Urbana Champaign, USA \\ \{scyan, huang\}ifp.uiuc.edu \\ ${ }^{2}$ Information Engineering Department, the Chinese University of Hong Kong, Hong Kong \\ $\{$ hwang5, jzliu, xtang\}ie.cuhk.edu.hk
}

\begin{abstract}
${ }^{1}$ The techniques for image analysis and classification generally consider the image sample labels fixed and without uncertainties. The rank regression problem is studied in this paper based on the training samples with uncertain labels, which is often the case for the manually estimated image labels. First, the core ranking model is designed as the bilinear fusing of multiple candidate kernels. Then, the parameters for feature selection and kernel selection are simultaneously learned by maximum a posteriori for given samples and uncertain labels. The convergency provable Expectation Maximization (EM) method is used for inferring these parameters in an iterative manner. The effectiveness of the proposed algorithm is finally validated by the extensive experiments on age ranking task and human tracking task. The popular FG-NET and the large scale Yamaha aging database are used for the age estimation experiments, and our algorithm significantly outperforms those state-of-the-art algorithms ever reported in literature. The experiment result on human tracking task also validates its advantage over conventional linear regression algorithm.
\end{abstract}

Keywords: Uncertain Label, Age Estimation, Human Tracking.

\section{INTRODUCTION}

Many image analysis tasks, e.g., age estimation and human object tracking, require to predict rank/ordinal labels of the data. This kind of tasks can be naturally formulated as general regression problems and solved with popular regression algorithms such as Multilayer Perceptrons (MLPs) [10]. In this work, we are interested in the ranking problems with uncertain labels, that is, the ordinal labels of the training samples are not fixed, but with known uncertainties and maybe characterized with specific intervals. In many real applications, it is often the case that we cannot exactly obtain the ordinal labels. For example, for age ranking problem, the exact age information is often difficult to obtain, but it is relatively much easier to obtain intervals to characterize the possible age ranges. Moreover, even the age of a certain person is labeled as a fixed value like 20 , the actual age can be any real value within the interval $\left[\begin{array}{ll}20 & 21\end{array}\right)$. Hence it makes more sense for the age to be finally expressed as an interval instead

\footnotetext{
${ }^{1}$ A short version of this paper appeared in ICME07.
} 


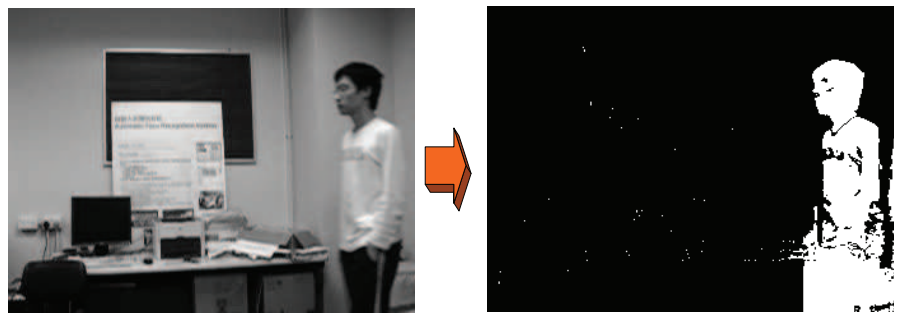

Fig. 1. Silhouette extraction from the video sequence.

of a fixed value. Several works [2][3][4][7] have been done to solve the age ranking problem, but none of them can solve the age ranking problem with uncertain labels.

For the problem of human tracking in videos, an interesting way for human tracking is to utilize person silhouette to predict the position of the human body [12]. For model training, we need manually label the positions of the human bodies in the videos. The extracted silhouette is often rough, and there may exist holes and incomplete areas, so the labeled positions of the human bodies may inevitably exist errors/uncertainties. Then, it is naturally valuable to design regressor which can effectively take the uncertain labels into consideration. Our work in this paper is dedicated to this specific target.

The contributions of this paper are two-fold. On the one hand, we propose a flexible ranking model by integrating the kernel trick and bilinear regression strategy. The kernel trick brings the potential to characterize the nonlinear relationship between the low-level features and high-level ordinal labels; and the bilinear regression strategy automatically selects the features and the way for kernel combination, which makes the algorithm flexible and adaptive for specific task. On the other hand, a general solution based Expectation-Maximization (EM) approach is proposed to solve the ranking problem with uncertain labels. Both the ranking model and solution approach are general for most ranking tasks, and the effectiveness of the whole framework is validated with the extensive experiments on age ranking and human tracking problem.

The rest of this paper is organized as follows. Section II introduces the motivations of this work. The details of the bilinear ranking model is discussed in Section III. Section IV provides the EM based solution to this problem, and the comparison experiments on two human age databases and the human tracking videos are presented in Section V. The concluding remarks are given in Section VI.

\section{Motivations}

The classification and regression are two fundamental problems for machine learning. Unlike the classification problems, the labels for regression task are often of real values, and consequently the labels may often be not so exact. For example, the measure of the height of a human, many nature and human factors may affect the final value. 
When specific to the problems of age estimation and human tracking, which we concern in this work, the labels are often not easy to obtain in an exact way. For age estimation, when we try to construct a database, the samples can be obtained in two ways. One is to ask the people to submit the images along with the age labels. In this scenario, the age is often given in an integer, like 20 , but the the actual age can be any real value within the interval $[20$ 21). The other way to collect a large set of face samples without age labels, and then ask many observers to label the ages. In this scenario, the labels of the same face image yet from different observers may be totally different. Commonly, the average of all age labels is used as the final ground truth, but obviously not all the information is well applied in the training stage. A natural way is to represent the age label as an interval, instead of a fixed value.

For human tracking problem as in [12], first a background model is constructed based on the mean and variance of each pixel in the empty background sequence. Then for the incoming video sequence, a pixel is considered as a background pixel if its Mahalanobis distance is no larger than a threshold, and the remaining are regarded as foreground pixels. Utilizing the background model, a rough personal silhouette is obtained as illustrated in Figure 1. The location of a person is considered as a function of the silhouette image difference between the current frame and a selected reference frame. Then regression algorithms are utilized for the estimation of the human position. For linear regression, the relationship of the human location $X$ and the silhouette image difference $D$ is assumed to be $X=R \times D$, where $R$ is the regression parameter matrix to be estimated in the training stage. As shown in Figure 1, the silhouette is not accurate enough, so it is very possible that there exist errors/uncertainties for the the manual labeled positions of human body. Similar to the age estimation problem, it is necessary to take into account these uncertainties within the labels for designing algorithm for human tracking.

In the following sections, we present our formulation and solution to the ranking problem with uncertain labels. This formulation and solution is general and can be used for solving any regression problem when the labels are manually labeled with possible uncertainties.

\section{BILINEAR RANKING MODEL}

As mentioned above, many image analysis problems, e.g., age ranking and human tracking, need predict the ordinal label information, and often there exists uncertainty within the obtained labels for the training samples. Here, we assume that the features extracted from the training samples (images or videos) are denoted as $X=$ $\left\{x_{1}, x_{2}, \ldots, x_{N}\right\}, x_{i} \in \mathbb{R}^{m}$, where $N$ is the sample number and $m$ is the feature dimension. The uncertain ordinal label for the sample $x_{i}$ is denoted as $\left[l_{i}^{\min }, l_{i}^{\max }\right]$, and the whole label set is denoted as $L=\left\{\left[l_{i}^{\min }, l_{i}^{\max }\right], i=\right.$ $1,2, \cdots, N\}$.

In this work, we integrate the kernel trick [9] and the bilinear regression strategy to build the ranking model, which maps from the low-level features to the high-level rank label. The model is motivated from the following 
observations: 1) the mapping from low-level features to high-level rank label is often nonlinear and the kernel trick offers the potential to uncover this kind of nonlinear relationship; and 2) how to select proper kernel functions is still an open problem, and the bilinear regression strategy provides a solution for automatic kernel selection along with the feature selection process.

Assume that we have a set of kernel mapping functions, denoted as $\left\{\phi^{1}(x), \phi^{2}(x), \cdots, \phi^{n}(x)\right\}$, where $n$ is the number of kernels, and $\phi^{o}(x): \mathbb{R}^{m} \rightarrow \mathfrak{F}^{o}, o=1,2, \cdots, n$, are the kernel mapping functions with $\mathfrak{F}^{o}$ as the higher or infinite dimensional Hilbert space and the corresponding kernel function $k^{o}(x, y)=<\phi^{o}(x), \phi^{o}(y)>$. Meanwhile, let the combined kernel mapping function be $\phi(x)=\left[\phi^{1}(x)^{T}, \phi^{2}(x)^{T}, \cdots, \phi^{n}(x)^{T}\right]^{T}$. It is obvious that the $k(x, y)=<\phi(x), \phi(y)>$ is also a kernel function.

The core idea of our proposed ranking model is to map $\phi(x)$ to the desired rank label. On the one hand, we utilize a linear regression model to map $\phi(x)$ to a scale value which targets at the desired rank label, and the projection direction is set as the linear combination of the mappings from all training samples, namely $\left[\phi\left(x_{1}\right), \phi\left(x_{2}\right)\right.$, $\left.\cdots, \phi\left(x_{N}\right)\right] u=\phi(X) u$ where $u \in \mathbb{R}^{N}$. One the other hand, we assign different weights to different kernel mapping functions for the sake of automatic kernel selection. The final bilinear ranking model is formally defined as

$$
l=(\phi(X) u)^{T}(\phi(x) . \times v)
$$

where $v \in \mathbb{R}^{n}$ is the weighting vector to give different weights for different kernels, and the operator.$\times$ is defined as

$$
\phi(x) . \times v=\left[\phi^{1}(x)^{T} v_{1}, \phi^{2}(x)^{T} v_{2}, \cdots, \phi^{n}(x)^{T} v_{n}\right]^{T} .
$$

Here, for each sample $x$, we define a data-specific kernel matrix $K_{x} \in \mathbb{R}^{N \times n}$ as

$$
K_{x}(j, o)=k^{o}\left(x_{j}, x\right)
$$

and for the training sample $x_{i}$, this kernel matrix is written as $K_{i}$. Then, Eqn. (1) can be rewritten as

$$
l=u^{T} K_{x} v
$$

An intuitive explanation of this ranking model is that the low-level feature vector $x$ is mapped into a matrix, and then two projection vectors are learned to bilinearly transform this data-specific matrix into the desired high-level rank label.

\section{EM-BASED PARAMETER LEARNING}

In this section, we introduce our procedure to learn the parameters $u$ and $v$ based on the training sample set $X$ and the corresponding uncertain label set $L$. 


\section{A. Likelihood Model}

Here, we assume that the data and their corresponding labels are independently sampled, and the likelihood model of $u$ and $v$ for given training sample set $X$ and label set $L$ is defined as

$$
p(X, L \mid u, v)=\prod_{i=1}^{N} \max _{l \in\left[l_{i}^{\text {min }}, l_{i}^{\text {max }}\right]} p\left(x_{i}, l \mid u, v\right) .
$$

Here, we have

$$
p\left(x_{i}, l \mid u, v\right) \propto \exp \left\{-\left\|l-u^{T} K_{i} v\right\|^{2} / \delta_{1}^{2}\right\}
$$

where $\delta_{1}$ is a constant and will be combined with another parameter $\theta_{2}$ as a single parameter $\lambda$ which is set experientially as described later.

\section{B. Prior Model}

To alleviate the possibility of overfitting to the training data, we present a prior model for the model parameters. Commonly, the norm of parameters is utilized for controlling overfitting. The larger is the norm, more possibly will the model overfit to the training data. Therefore, a reasonable prior model for the model parameters is

$$
p(u, v) \propto \exp \left\{-\left(\|u\|^{2}+\|v\|^{2}\right) / \delta_{2}^{2}\right\},
$$

where the parameter $\delta_{2}$ is a constant.

\section{Maximum a Posteriori}

Based on the above likelihood model and the prior model, the ranking model parameters are derived by maximum a posteriori as

$$
\arg \max _{u, v}\{p(u, v \mid X, L) \propto p(u, v) p(X, L \mid u, v)\} .
$$

There does not exist closed-form solution for maximum a posteriori due to the maximization operator in the likelihood model. In this work, we consider this optimization problem as an incomplete data problem. The missing data is the desired rank label, denoted as $l_{i}$ for sample $x_{i}$, and the desired label set is $L^{m}=\left[l_{1}, l_{2}, \cdots, l_{N}\right]$. The complete-data model is

$$
p\left(u, v \mid X, L, L^{m}\right)=p\left(u, v \mid X, L^{m}\right) \propto p(u, v) p\left(X, L^{m} \mid u, v\right),
$$

where

$$
p\left(X, L^{m} \mid u, v\right) \propto \prod_{i=1}^{N} \exp \left\{-\left\|l_{i}-u^{T} K_{i} v\right\|^{2} / \delta_{1}^{2}\right\} .
$$




\section{Parameter estimation with EM}

Here, we use the Expectation Maximization (EM) algorithm [11] for parameter estimation with incomplete data. Denote the parameter estimate obtained at the $n$-th step by $\theta_{n}=\left\{u_{n}, v_{n}\right\}$. The $Q$-function [11] can then be obtained as

$$
Q\left(\theta \mid \theta_{n}\right)=E_{L^{m}}\left\{p\left(\theta \mid X, L, L^{m}\right) \mid X, L, \theta_{n}\right\}
$$

where the expectation is taken w.r.t. $p\left(L^{m} \mid X, L, \theta_{n}\right)$.

1) E-Step: For given $X, L$ and $\theta_{n}$, the desired rank label for a certain data is fixed, namely with probability of 1 for a value while probability of 0 for all other values. More specifically speaking, the desired rank label of sample $x_{i}$ has probability of 1 to be

$$
\tilde{l}_{i}^{m}= \begin{cases}l_{i}^{\min }, & \text { if } u_{n}^{T} K_{i} v_{n}<l_{i}^{\min } \\ l_{i}^{\max }, & \text { if } u_{n}^{T} K_{i} v_{n}>l_{i}^{\max } \\ u_{n}^{T} K_{i} v_{n}, & \text { else }\end{cases}
$$

Denote $\tilde{L}^{m}=\left[\tilde{l}_{1}^{m}, \tilde{l}_{2}^{m}, \cdots, \tilde{l}_{N}^{m}\right]$, then we have

$$
p\left(L^{m} \mid X, L, \theta_{n}\right)=\left\{\begin{array}{l}
1, \text { if } L^{m}=\tilde{L}^{m} \\
0, \text { else. }
\end{array}\right.
$$

Consequently, the $Q$-function can be simplified as

$$
Q\left(\theta \mid \theta_{n}\right)=p\left(\theta \mid X, \tilde{L}^{m}\right)
$$

2) M-Step: In the M-step, we maximize the $Q$-function

$$
\begin{array}{r}
Q\left(\theta \mid \theta_{n}\right) \propto \exp \left\{-\left(\|u\|^{2}+\|v\|^{2}\right) / \delta_{2}^{2}\right\} \times \\
\prod_{i=1}^{N} \exp \left\{-\left\|\tilde{l}_{i}^{m}-u^{T} K_{i} v\right\|^{2} / \delta_{1}^{2}\right\}
\end{array}
$$

which is equivalent to minimize

$$
F(u, v)=\left(\|u\|^{2}+\|v\|^{2}\right) / \delta_{2}^{2}+\sum_{i=1}^{N}\left\|\tilde{l}_{i}^{m}-u^{T} K_{i} v\right\|^{2} / \delta_{1}^{2}
$$

The objective function is quartic and commonly there does not exist closed-form solution. In this work, we present an iterative solution to search for the local optimum.

For given $u$, we set the derivative of $F(u, v)$ w.r.t $v$ as zero to obtain the corresponding optimal value of $v$, namely

$$
\frac{\partial F(u, v)}{\partial v}=\frac{2}{\delta_{2}^{2}} v-\sum_{i=1}^{N} \frac{2}{\delta_{1}^{2}}\left(u^{T} K_{i}\right)^{T}\left(\tilde{l}_{i}^{m}-u^{T} K_{i} v\right)=0
$$

Then, we have

$$
v=\left(\lambda I+\sum_{i=1}^{N} K_{i}^{T} u u^{T} K_{i}\right)^{-1} \sum_{i=1}^{N} K_{i}^{T} u \tilde{l}_{i}^{m}
$$




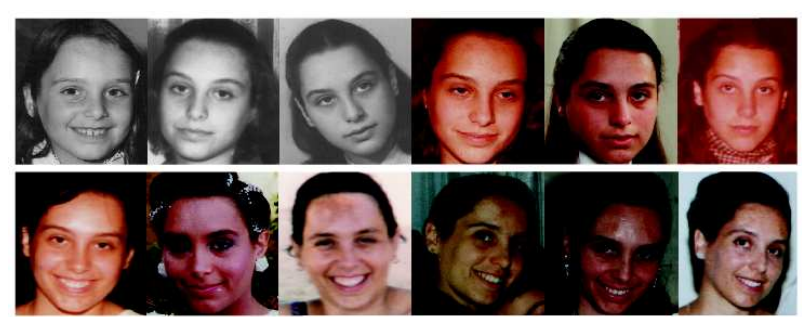

Fig. 2. Sample aging images from one person in FG-NET Aging Database.

where $\lambda=\delta_{1}^{2} / \delta_{2}^{2} . \lambda$ is the parameter to balance two terms on prior model and likelihood model, and in this paper, $\lambda$ is experientially set as 0.0005 in all the experiments.

Similarly, for given $v$, we set the derivative of $F(u, v)$ w.r.t $u$ as zero to obtain the corresponding optimal value of $u$, namely

$$
\frac{\partial F(u, v)}{\partial u}=\frac{2}{\delta_{2}^{2}} u-\sum_{i=1}^{N} \frac{2}{\delta_{1}^{2}}\left(v^{T} K_{i}^{T}\right)^{T}\left(\tilde{l}_{i}^{m}-u^{T} K_{i} v\right)=0 .
$$

Then, we have

$$
u=\left(\lambda I+\sum_{i=1}^{N} K_{i} v v^{T} K_{i}^{T}\right)^{-1} \sum_{i=1}^{N} K_{i} v \tilde{l}_{i}^{m},
$$

We iteratively optimize $u$ and $v$ until the norms of differences between the solutions of two successive steps are both smaller than a manually set threshold (set as $10^{-4}$ in this work), or after a predefined number of loops (set as 20 in this work). Meanwhile, the whole algorithm iterates between the E-step and M-step until converged.

When a new datum $\mathrm{x}$ comes, we first compute the data-specific kernel matrix $K_{x}$ as in Eqn. (3), and then its ordinal label is predicted as in Eqn. (4).

\section{EXPERIMENTS}

In this section, we take the age ranking and human tracking problems as examples to illustrate the effectiveness of our proposed algorithm for ranking with uncertain labels.

\section{A. Experiment Configurations}

Two aging face databases are used in our experiments on age estimation. One is the FG-NET aging database [1], which contains 1002 face images of 82 persons with ages ranging from 0 to 69 . Some sample images are displayed in Figure 2. The evaluation framework for the FG-NET database is the Leave-One-Person-Out (LOPO). The other data set Yamaha face database ${ }^{2}$ contains 800 males and 800 females, and 8000 images with ages ranging from 0 to 93 . The experiments are carried out separately on female and male subsets respectively. For each subset, the images are randomly divided into 4 folds, and 4-fold cross-validation is performed for the evaluation of different algorithms.

\footnotetext{
${ }^{2}$ To protect the portrait rights of the participants, sample images of the Yamaha face database are not shown here.
} 


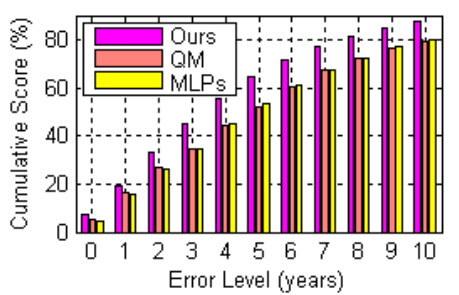

(a) FG-NET.

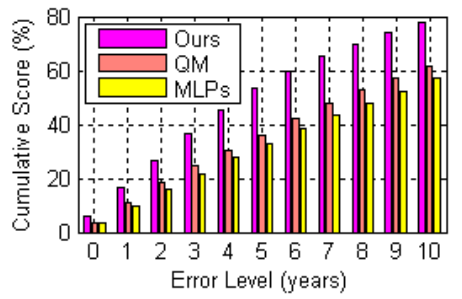

(b) Female@YAMAHA.

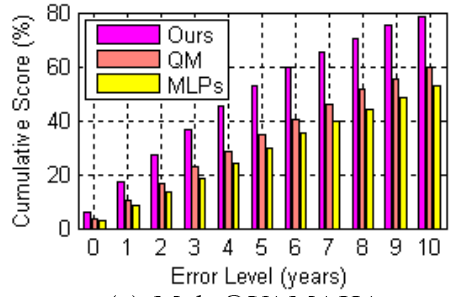

(c) Male@YAMAHA.

Fig. 3. Cumulative scores for Quadratic Model, MLPs and our algorithm at absolute error levels from 0 to 10 years.

In the FG-NET database, each person has multiple images of different ages, and hence it is relatively easy to extract features characterizing aging process; while for the Yamaha database, the images of the same person are of the same age, and hence many algorithms such as aging patterns subspace (AGES) [7] and Weighted Appearance Specific (WAS) [8] are inapplicable due to their requirement of multiple images of different ages for each person.

For comparison, we use the same feature set as in [7] for the FG-NET database, and the first 200 appearance parameters [6] based on the 68 key facial points are used as input for age ranking. For detailed information on shape, texture and appearance parameters, please refer to [5]. For the Yamaha database, as we do not have the positions of the key facial points as in the FG-NET database, the face images are cropped and normalized to size of 64 -by- 64 pixels by fixing the locations of the two eyes. The original gray level values are used as features for age ranking.

For the human tracking problem, we captured two video sequences at two sessions, and one frame is shown as in Figure 1. We manually labeled all the positions of the human body. We use the first session with 59 frames for model training and the other session with 89 frames for testing. The person movement is fixed on a line and no scale variation occurred so that the location to be estimated is a scalar. The reference frame is the one where the human is roughly in the center of the image.

\section{B. Age Ranking Results}

As mentioned beforehand, the age information is often present as an integer, but the age can actually be any real value. For example, when we say a person is of age $n$, its real age can be any value within $[n, n+1)$. In this paper, we set the age label as $[n, n+1-\epsilon]$ for our algorithm, where $\epsilon$ is the smallest positive value that a computer can encode.

The following two algorithms are systematically compared with our algorithm. In [4], the relationship between the age labels and the feature vectors is modeled as a quadratic regression model (QM), namely,

$$
a=c+w_{1}^{T} x+w_{2}^{T}\left(x^{2}\right),
$$

where $x$ and $\left(x .^{2}\right)$ are the vectors containing the features and the squares of the features respectively, $c, w_{1}$, and $w_{2}$ are parameters. Another popular regression algorithm is Multilayer Perceptrons (MLPs) with the back propagation 
TABLE I

MAES OF DIFFERENT ALGORITHMS ON FG-NET AND YAMAHA AGING DATABASES. NOTE THAT AGES WAS THE BEST ALGORITHM EVER REPORTED ON THE FG-NET DATABASE.

\begin{tabular}{|c||c||c|c|}
\hline Algorithm & FG-NET & Female@ YA & Male@ YA \\
\hline \hline WAS [7] & 8.06 & N/A & N/A \\
\hline AGES [7] & 6.77 & N/A & N/A \\
\hline QM & 6.55 & 9.96 & 10.51 \\
\hline MLPs & 6.98 & 10.99 & 12.0 \\
\hline Ours & $\mathbf{5 . 3 3}$ & $\mathbf{6 . 9 5}$ & $\mathbf{6 . 9 5}$ \\
\hline
\end{tabular}

learning [10].

In both databases, the gaussian kernels $k_{o}(x, y)=\exp \left\{-\|x-y\|^{2} / \delta_{o}^{2}\right\}$ are applied as the kernel candidates and we use 4 kernels with parameters $\delta_{o}=2^{(o-10) / 2.5} \delta, o=0,1,2,3$ in all the experiments, where $\delta$ is the standard deviation of the sample data.

Two measures are used to evaluate algorithmic performance. The first one is the Mean Absolute Error (MAE) criterion used in [4][8][7]. MAE is defined as an average of the absolute errors between the estimated ages and the ground truth ages, i.e., MAE $=\sum_{i=1}^{N_{t}}\left|\hat{l}_{i}-l_{i}\right| / N_{t}$, where $\hat{l}_{i}$ is the estimated age for the $i$-th sample, $l_{i}$ is the ground truth age for the testing images and $N_{t}$ is the number of testing images. Another popular measure is the cumulative score [7]: $C S(\theta)=N_{e \leq \theta} / N_{t} \times 100 \%$, where $N_{e \leq \theta}$ is the number of samples on which the estimator makes an absolute error no higher than $\theta$.

A detailed comparison of the age estimation accuracy is displayed in Figure 3 and listed in Table I. Table I lists the MAEs of different algorithms on both FG-NET and Yamaha databases. From these results, we can have the observations: 1) our algorithm significantly outperforms the state-of-the-art age ranking algorithms which consider the age labels fixed; and 2) AGES is the best algorithm ever reported for age ranking, and it performs better than MLPs and WAS, yet worse than QM in our experiment. A possible explanation is that many aging patters are incomplete in FG-NET database.

\section{Human Tracking Results}

For our proposed algorithm, 20 gaussian kernels kernels $k_{o}(x, y)=\exp \left\{-\|x-y\|^{2} / \delta_{o}^{2}\right\}$ are used with parameters $\delta_{o}=2^{(o) / 2.5} \delta, o=0,1, \ldots, 20$, where $\delta$ is the standard deviation of the sample data. The regression interval is set as $10(+/-5)$ pixels for our proposed algorithm. We compared our algorithm with the linear regression algorithm as described beforehand. The cumulative score for the testing sequence is shown in Figure 4, which again validates the effectiveness of our proposed algorithm.

\section{CONCLUSIONS}

In this paper, we proposed a general formulation and solution for regression problem with uncertain labels. The bilinear regression model was presented for modeling the nonlinear relationship between the low-level image 


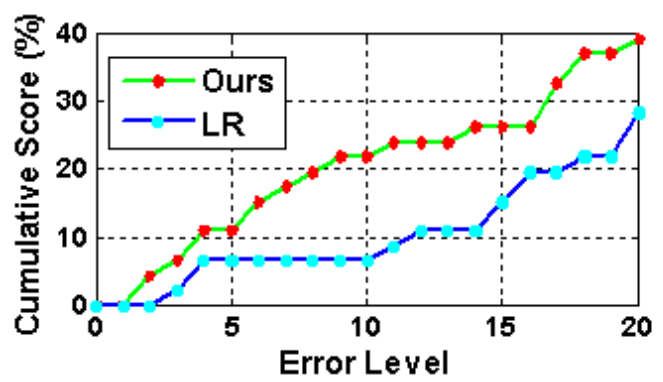

Fig. 4. Cumulative score of the human tracking results. The overall MAE: 15.6673 pixels (ours) versus 20.9172 pixels (Linear Regression).

features and the high-level labels. The EM-based solution well utilized the uncertain information and encouraging experimental results were achieved in both age ranking and human tracking problems.

\section{REFERENCES}

[1] The FG-NET aging database: http://sting.cycollege.ac.cy/ alanitis/fgnetaging/index.htm.

[2] J. Hayashi, M. Yasumoto, H. Ito and H. Koshimizu, "A method for estimating and modeling age and gender using facial image processing," Seventh International Conference on Virtual Systems and Multimedia, pp. 439-448, 2001.

[3] Y. Kwon and N. Lobo, "Age classification from facial images," Computer Vision and Image Understanding, vol. 74, no. 1, pp. 1-21, 1999.

[4] A. Lanitis, C. Draganova and C. Christodoulou, "Comparing different classifiers for automatic age estimation," vol. 34, no. 1, pp. 621-628, February 2004.

[5] T. Cootes, G. Edwards and C. Taylor, "Active appearance models," IEEE Transactions on Pattern Analysis and Machine Intelligence, vol. 23, no. 6, pp. 681-685, 2001.

[6] G. Edwards and A. Lanitis. Statistical face models: Improving specificity. Image and Vision Computing, vol. 16, no. 3, pp. 203-211, 1998.

[7] X. Geng, Z. Zhou, Y. Zhng, G. Li, and H. Dai, "Learning from facial aging patterns for automatic age estimation," Proceedings of ACM Multimedia'06, 2006.

[8] A. Lanitis, C. Taylor and T. Cootes, "Toward automatic simulation of aging effects on face images," IEEE Transactions on Pattern Analysis and Machine Intelligence, vol. 24, no. 4, pp. 442-455, 2002.

[9] K. Müller, S. Mika, G. Rätsch, K. Tsuda, and B. Schölkopf, "An introduction to kernel-based learning algorithms," IEEE Transactions on Neural Networks, vol. 12, no. 2, pp. 181-202, 2001.

[10] G. Hinton, D. Rumelhart and R. Williams, "Learning representations by back-propagating errors," Nature, vol. 323, pp. 533-536, 1986.

[11] J. Bilmes. A gentle tutorial of the EM algorithm and its application to parameter estimation for Gaussian mixture and hidden Markov models. Technical report, International Computer Science Institute, Berkeley, 1998.

[12] K. Rerkrai and H. Fillbrandt. Tracking Persons under Partial Scene Occlusion Using Linear Regression. The Eighth International Student Conference on Electrical Engineering, 2004. 